\title{
Video-Assisted Thoracoscopic Surgery and Minimal Access Spinal Surgery Compared in Anterior Thoracic or Thoracolumbar Junctional Spinal Reconstruction: A Case-Control Study and Review of the Literature
}

\author{
Ching-Yu Lee, ${ }^{1,2,3}$ Meng-Huang Wu, ${ }^{3,4,5}$ Yen-Yao Li, ${ }^{1,2}$ Chin-Chang Cheng, ${ }^{1,2,3}$ \\ Chien-Yin Lee, ${ }^{1}$ and Tsung-Jen Huang ${ }^{4,5}$ \\ ${ }^{1}$ Department of Orthopedic Surgery, Chang Gung Memorial Hospital, Chiayi, Taiwan \\ ${ }^{2}$ College of Medicine, Chang Gung University, Taoyuan, Taiwan \\ ${ }^{3}$ Graduate Institute of Clinical Medical Sciences, College of Medicine, Chang Gung University, Taoyuan, Taiwan \\ ${ }^{4}$ Department of Orthopedic Surgery, Taipei Medical University Hospital, Taipei, Taiwan \\ ${ }^{5}$ Department of Orthopedics, School of Medicine, College of Medicine, Taipei Medical University, Taipei, Taiwan \\ Correspondence should be addressed to Tsung-Jen Huang; tjdhuang@tmu.edu.tw
}

Received 31 July 2016; Revised 16 November 2016; Accepted 1 December 2016

Academic Editor: William B. Rodgers

Copyright (C) 2016 Ching-Yu Lee et al. This is an open access article distributed under the Creative Commons Attribution License, which permits unrestricted use, distribution, and reproduction in any medium, provided the original work is properly cited.

\begin{abstract}
There are no published reports that compare the outcomes of video-assisted thoracoscopic surgery (VATS) and minimal access spinal surgery (MASS) in anterior spinal reconstruction. We conducted a retrospective case-control study in a single center and systematically reviewed the literature to compare the efficacy and safety of VATS and MASS in anterior thoracic (T) and thoracolumbar junctional (TLJ) spinal reconstruction. From 1995 to 2012, there were 111 VATS patients and 76 MASS patients treated at our hospital. VATS patients had significantly $(p<0.001)$ longer operating times and significantly $(p<0.022)$ higher thoracotomy conversion rates. We reviewed 6 VATS articles and 10 MASS articles, in which there were 625 VATS patients and 399 MASS patients. We recorded clinical complications and a thoracotomy conversion rate from our cases and the selected articles. The incidence of approach-related complications was significantly $(p=0.021)$ higher in VATS patients. The conversion rate was $2 \%$ in VATS patients and $0 \%$ in MASS patients $(p=0.001)$. In conclusion, MASS is associated with reduction in operating time, approach-related complications, and the thoracotomy conversion rate.
\end{abstract}

\section{Introduction}

Video-assisted thoracoscopic surgery (VATS) and minimal access spinal surgery (MASS) have been considered primarily as minimally invasive surgery (MIS) for anterior thoracic (T) and thoracolumbar junction (TLJ) spine surgery [1]. VATS was first described by Mack et al. in 1993 [2]; it allows for biopsy, anterior release, abscess drainage, and discectomy [3, 4]. VATS has been used to treat anterior thoracic diseases at our hospital since 1995. Over the next 10 years, we used VATS in many spinal procedures: decompression, corpectomy, reconstruction, and stabilization. The microsurgical miniopen anterolateral approach was first introduced in 1997 by
Mayer [5] for minimally invasive anterior lumbar interbody fusion. Kossmann et al. [6] reported in 2001 that the anterior column of the thoracic spine could easily be assessed and reconstructed using a minithoracotomy and a table-mounted retractor. At that time, we developed a new VATS approach [7-10], which we called the "extended manipulating channel method." It allowed us to use a combination of conventional spinal instruments and VATS to enter the chest cavity and to manipulate those instruments as we would for standard open surgical procedures. Furthermore, at our hospital, a refined MASS has been evolving since 2000 from our extended manipulating channel method without VATS [11, 12]. MASS has been used to treat vertebral metastasis, osteomyelitis, and 
fractures. It is generally believed that because MASS allows direct three-dimensional vision of the surgical field, which seems to make the procedure familiar to spine surgeons used to standard open surgical procedures, it has become more popular than VATS. Thus, we compared the outcomes of MASS in anterior T and TLJ spinal reconstruction and fusion with those of VATS.

\section{Patients and Methods}

2.1. Patients. We identified, in our hospital's Spine Operation Registry, all patients who underwent VATS (Figures 1 and 2) and MASS (Figure 3). We previously published reports which described both VATS [7, 8, 10, 13] and MASS [12, 14] techniques for anterior $\mathrm{T}$ or TLJ spinal reconstruction between 1995 and 2012 and retrospectively reviewed their records. The inclusion criteria were anterior intervertebral fusion after a discectomy with a partial or a total corpectomy for treating spinal fractures, vertebral malignancy, infectious spondylitis, thoracic disc herniation, and degenerative spinal diseases. Patients with pediatric scoliosis, a discectomy without fusion, or a biopsy were excluded from the study. All included patients had undergone minimally invasive anterior spine reconstruction performed by one senior surgeon ( $\mathrm{T}$. J. Huang). We reviewed the patients' medical records and recorded data on operating time, estimated blood loss, need for intensive care, conversion to standard open thoracotomy, and complications in patients with T and TLJ spinal disorders. Approval for this study was obtained from the Ethics Committee and Institutional Review Board of our hospital (IRB number 101-1238B).

2.2. Review of Published Literature. The English language literature published between 1995 and 2012 was systematically reviewed. The Cochrane Review Database, EMBASE, Medline, PubMed, and Google Scholar were searched. The reference lists of the selected articles were checked. Search terminology included miniopen, MASS, VATS, anterior $T$ spinal surgery, TLJ (T11-L2) spinal surgery, and anterior spinal fusion. We excluded studies associated with pediatric spine surgery, disc excision without fusion, and anterior lumbar surgery (L3-L5). Technical notes, case reports, anatomical descriptions, or a combined surgery of thoracoscopic surgery and thoracotomy was not included. The articles were screened and selected by two independent reviewers (Y. Y. $\mathrm{Li}$ and C. C. Cheng) based on the inclusion and exclusion criteria. Disagreements were resolved by discussion or by a consultation with a third reviewer (Ching-Yu Lee). The data of the selected articles were extracted and analyzed in detail by two independent reviewers (M. H. Wu and Chien-Yin Lee). Because data on the surgical complications were going to be analyzed, the interrater agreement about these data was analyzed using the kappa statistic. Disagreements were resolved by discussion or by a consultation with a senior spine surgeon (T. J. Huang).

2.3. Data Analysis. The perioperative parameters of our included sample were operating time, estimated blood loss, complications, conversion to standard thoracotomy, and the need for postoperative admission to the intensive care unit. They were recorded and compared between our VATS and MASS patients. The perioperative data of the selected articles were average operating time, average estimated blood loss, complication rates, and conversions to thoracotomy.

Data of clinical complications and conversions to standard thoracotomy, which were recorded from our cases and the selected articles, were compared between VATS and MASS patients. A minor complication was defined as a minor risk event with no treatment, with medical treatment, or with intraoperative repair but without long-term sequelae. A major complication was defined as a life-threatening or irreversible event requiring invasive treatment or revision surgery. Death was mortality because of associated perioperative complications.

An approach-related complication was defined as intercostal neuralgia, pleural effusion, or air leakage causing subcutaneous emphysema or pneumothorax $[15,16]$.

\section{Statistical Methods}

All statistical analyses were done using SPSS 12.0 for Windows. An independent Student $t$-test was used for numerical data. An $\chi^{2}$ analysis or a Fisher exact test was used for categorical data. Significance was set at $p<0.05$. The observed interrater agreement for the data extracted from the selected publications was analyzed using the kappa statistic.

\section{Results}

We reviewed the medical records of 187 patients who had undergone minimally invasive surgery (MIS) for anterior T or TLJ spinal fusion at our hospital between 1995 and 2012. VATS was used in 111 patients, and MASS was used in the other 76 patients (Table 1). Operating time was longer in the VATS group than in the MASS group $(p<0.001)$. There was a significantly higher incidence of conversion to standard open thoracotomy in the VATS group than in the MASS group $(p=0.022)$. There were no significant differences in average blood loss or the need for postoperative admission to the intensive care unit (ICU).

\section{Literature-Reported Results}

There were 16 articles about MIS for anterior T/TLJ spinal fusion (Table 2): 6 VATS articles [17-22] and 10 MASS articles [6, 23-31]. Of the 6 VATS articles, the median average operating time was 223 minutes (range: $155-347$ minutes), the median average estimated blood loss was $585 \mathrm{~mL}$ (range: 310 $1117 \mathrm{~mL}$ ), the median complication rate was $25.9 \%$ (range: 9.4-34\%), and the median conversion rate was $0.5 \%$ (range: $0-6.2 \%)$. Of the 10 MASS articles, the median average operating time was 170 minutes (range: 101-210 minutes), the median average estimated blood loss was $423 \mathrm{~mL}$ (range: 290$912 \mathrm{~mL}$ ), the median complication rate was $14.9 \%$ (range: $0-33 \%)$, and there were no conversions to standard open procedure.

Perioperative complications were collected from 187 patients of our institute and 1024 patients of the 16 selected 


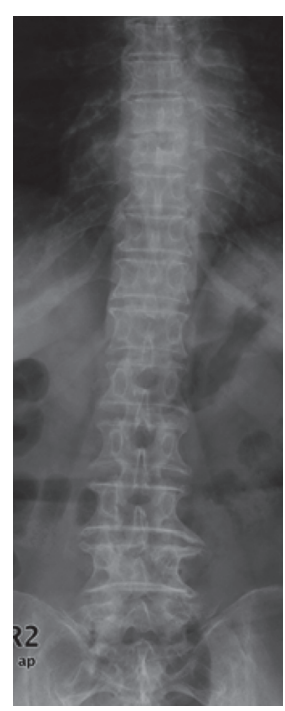

(a)

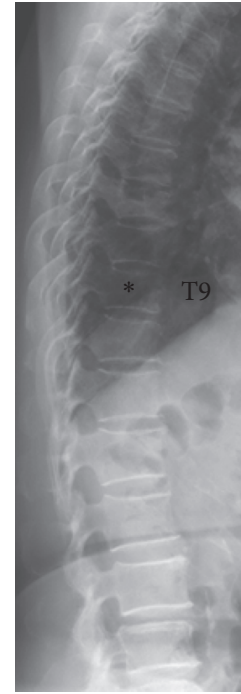

(b)

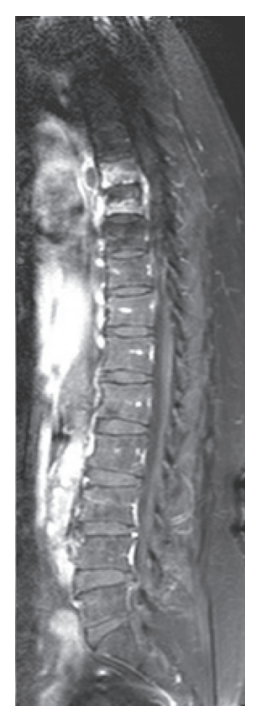

(c)

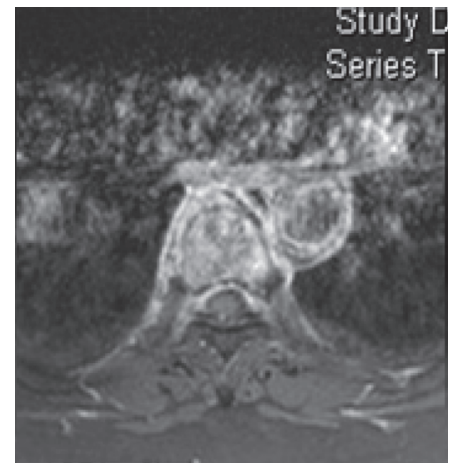

(d)

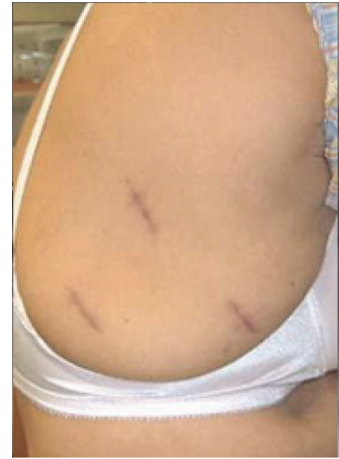

(e)

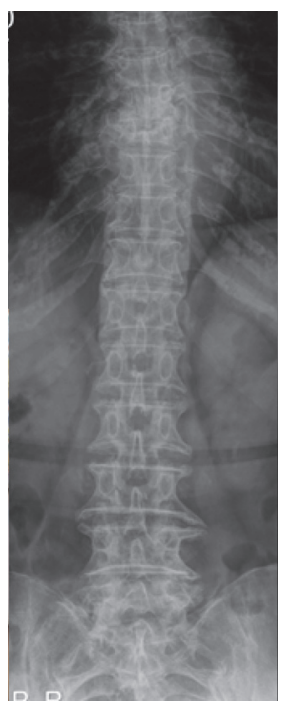

(f)

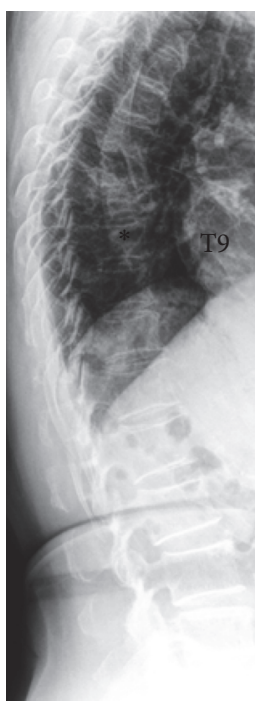

$(\mathrm{g})$

FiguRE 1: Video-assisted thoracoscopic surgery (VATS) for treating tuberculous spondylitis of T7-8 in a 74-year-old woman. (a) and (b) Vertebral destruction and collapse in T8. (c) and (d) Gadolinium-enhanced magnetic resonance imaging (MRI) shows osteomyelitis in T7-8 vertebral bodies and anterior epidural abscess spreading under the anterior longitudinal ligament. (e) The incisional wound was $2.5-3.0 \mathrm{~cm}$ long to allow a three-portal video-assisted thoracoscopic debridement, curettage, and harvested tricortical iliac strut bone graft for anterior spinal reconstruction on T7-8. (f) and (g) Solid bone fusion was noticed on T7-8 at the 2-year follow-up. 


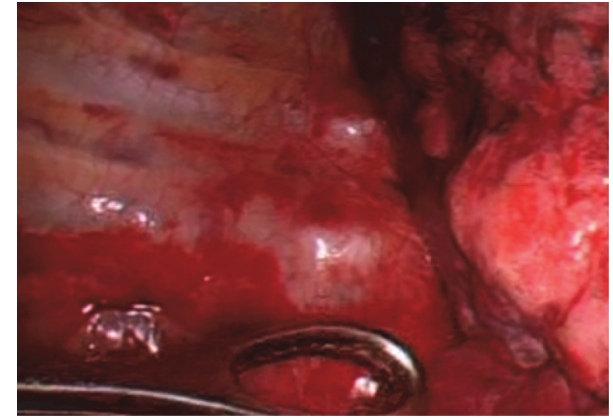

(a)

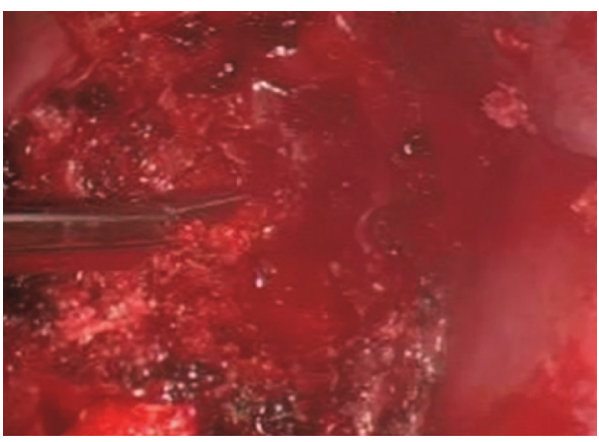

(c)

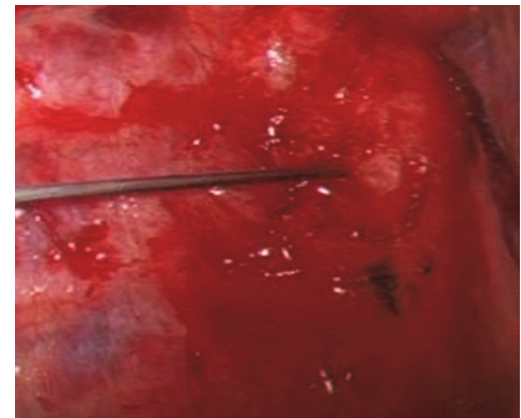

(b)

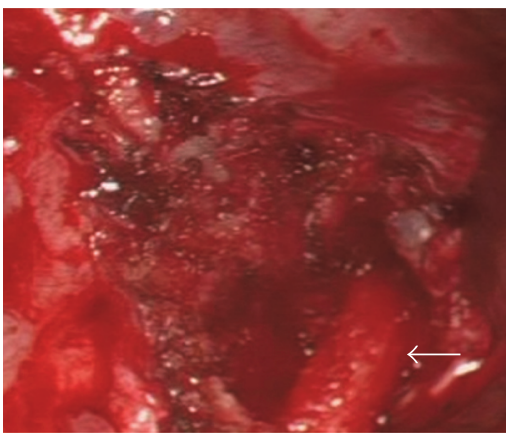

(d)

FIGURE 2: Video-assisted thoracoscopic surgery (VATS) spinal approach to tuberculous spondylitis of T7-8. (a) and (b) The lesion site was identified using fluoroscopy and was displayed on the video monitor. The lesion site was initially covered with the visceral pleura because of inflammation. (c) The infected vertebral body and soft tissue were removed using pituitary rongeurs and elongated curettes. (d) Column reconstruction with intervertebral fusion was initiated using an autogenous tricortical iliac strut graft (white arrow).

TABLE 1: MIS for anterior T and TLJ spinal reconstruction in 187 patients at our Institution.

\begin{tabular}{|c|c|c|c|}
\hline & VATS & MASS & $p$ value \\
\hline Number of patients & 111 & 76 & \\
\hline Male/female & $68 / 43$ & $39 / 37$ & 0.177 \\
\hline Mean age (year) & $57.1 \pm 14.5$ & $60.4 \pm 14.8$ & 0.133 \\
\hline Number of pathologic regions & & & 0.085 \\
\hline $\mathrm{T}$ & $59(53)$ & $50(66)$ & \\
\hline TLJ & $52(47)$ & $26(34)$ & \\
\hline Number of pathologic types & & & 0.253 \\
\hline Fracture & $25(23)$ & $9(12)$ & \\
\hline Infectious spondylitis & $31(28)$ & $24(32)$ & \\
\hline Spinal malignancy & $49(44)$ & $36(47)$ & \\
\hline Disc herniation or degeneration & $6(5)$ & $7(9)$ & \\
\hline \multicolumn{4}{|l|}{ Perioperative data } \\
\hline Operating time ${ }^{\#}$ (mins) & $224.5 \pm 68.6$ & $183.5 \pm 33.2$ & $<0.001^{*}$ \\
\hline Estimated blood loss ${ }^{\#}(\mathrm{ml})$ & $916.0 \pm 660.3$ & $933.8 \pm 847.6$ & 0.879 \\
\hline Conversion to standard thoracotomy & $8(7)$ & 0 & $0.022^{*}$ \\
\hline Need for postoperative ICU care & $9(8)$ & $4(5)$ & 0.565 \\
\hline
\end{tabular}

Data are expressed as mean \pm standard deviation or number $(\%) .{ }^{*} p<0.05$.

${ }^{\#}$ Patients undergoing conversion thoracotomy were not included.

MIS: minimally invasive surgery; VATS: video-assisted thoracoscopic surgery; MASS: minimal access spinal surgery; T: thoracic; TLJ: thoracolumbar junction; ICU: intensive care unit. 


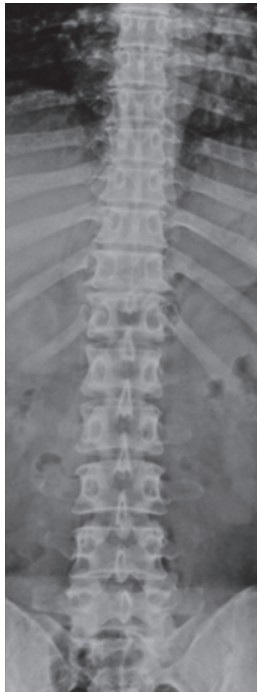

(a)

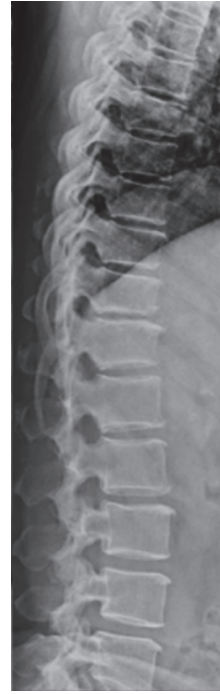

(b)

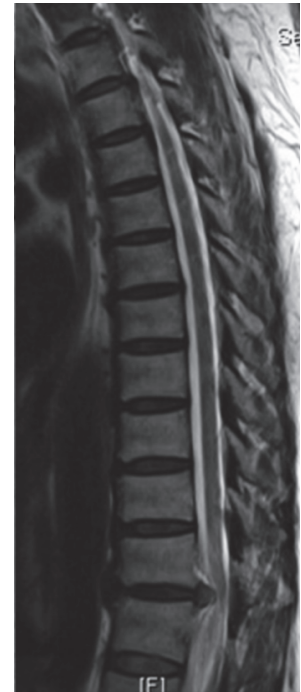

(c)

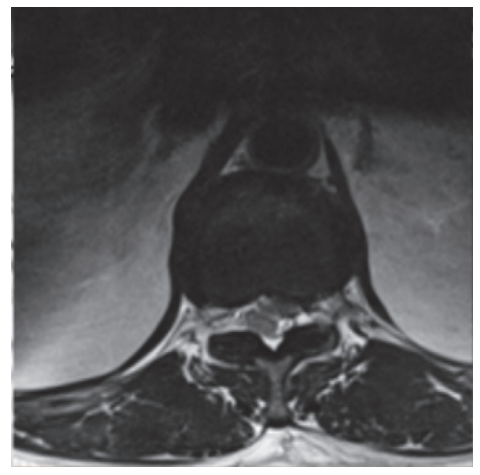

(d)

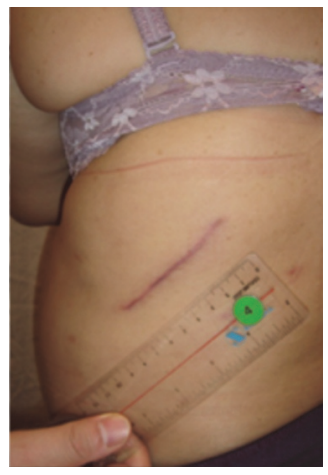

(e)

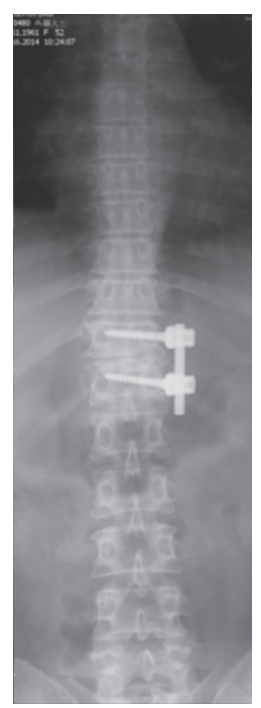

(f)

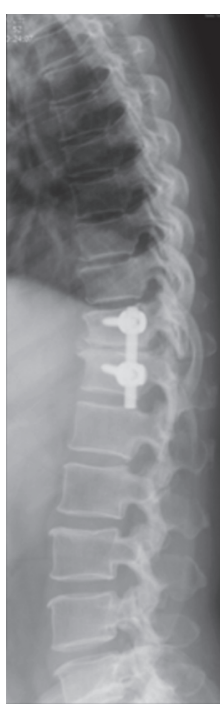

(g)

FIGURE 3: Anterior minimal access spinal surgery for treating thoracic disc herniation of T11-12 in a 41-year-old woman. (a) and (b) Narrowing disc space with endplate sclerosis on T11-12 level was noticed. (c) and (d) Magnetic resonance imaging (MRI) shows left paracentral disc herniation on T11-12 level. (e) A $7 \mathrm{~cm}$ skin incision in the patient's left lateral thoracic cage. (f) and (g) Anterior retropleural and retroperitoneal approach for thoracic discectomy and fusion was performed using a double-barreled rib strut graft and anterior vertebral instrumentation. No intraoperative one-lung ventilation, a postoperative chest tube, or ICU care was given. Solid bone fusion on T11-12 was noticed at the 2-year follow-up. 
TABLE 2: A literature review of MIS for anterior T and TLJ spinal reconstruction.

\begin{tabular}{lccccccc}
\hline Authors & Years & PT no. & AOT (min) & ABL (ml) & CR (\%) & TCR (\%) \\
\hline Dickman et al. & 1996 & 17 & VATS for reconstruction in T spine & 347 & 117 & 29.4 & 0 \\
Khoo et al. & 2002 & 371 & VATS in treating T or TL spinal fractures & 240 & 650 & 9.7 & 1.1 \\
Kapoor et al. & 2005 & 16 & VATS in treating TB spondylitis & 223 & 497 & 31.2 & 6.2 \\
Le Huec et al. & 2010 & 50 & VATS for treating TLJ fractures & 155 & 620 & 20.0 & 0 \\
Lü et al. & 2012 & 50 & VATS in treating thoracic TB spondylitis & 210 & 550 & 34.0 & 0 \\
Wait et al. & 2012 & 121 & VATS for discectomy and fusion in T spine & NA & 310 & 22.3 & 1.7 \\
Kossmann et al. & 2001 & 58 & MASS for reconstruction in T/TLJ(58) + L(7) & 170 & 912 & $7.7 \%$ & 0 \\
El Saghir & 2002 & 21 & MASS for reconstruction in TL spine & 101 & 724 & $33 \%$ & 0 \\
Scheufler & 2007 & 38 & MASS for reconstruction in T/TLJ spine & 167 & 652 & $18 \%$ & 0 \\
Payer and Sottas & 2008 & 37 & MASS for reconstruction in TL spine & 181 & 632 & $16.2 \%$ & 0 \\
Smith et al. & 2010 & 52 & MASS in treating TLJ fractures & 117 & 300 & $13.5 \%$ & 0 \\
Uribe et al. & 2010 & 21 & MASS in treating T spinal tumor & 188 & 423 & $4.8 \%$ & 0 \\
Khan et al. & 2012 & 20 & MASS for reconstruction in T/TLJ(20) + L(4) & 291 & 0 \\
Deviren et al. & 2011 & 12 & MASS for reconstruction in T spine & 210 & 400 & $16.7 \%$ & 0 \\
Baaj et al. & 2012 & 80 & MASS for reconstruction in TL spine & NA & NA & $12.5 \%$ & 0 \\
Uribe et al. & 2012 & 60 & MASS for discectomy and fusion in T spine & 182 & 290 & $25 \%$ \\
\hline
\end{tabular}

MIS: minimally invasive surgery; T: thoracic; TLJ: thoracolumbar junction; PT no.: patient number; AOT: average operating time; ABL: average estimated blood loss; CR: complication rate; TCR: thoracotomy conversion rate; VATS: video-assisted thoracoscopic surgery; MASS: minimal access spinal surgery.

publications (Table 3). The assessment score agreement between the reviewers was good (kappa statistic: $0.62, p<$ $0.001)$. There were $126(17 \%)$ perioperative complications in VATS patients and $71(15 \%)$ in MASS patients $(p=$ $0.317)$ : there was not significantly different distribution of no, minor, and major complication $(p=0.567)$. Revision surgery was the most common major complication in both groups: 11 VATS patients and 8 MASS patients. There were 6 mortalities in this study, 3 in each cohort of VATS and MASS: 1 with pneumonia, 1 with acute thromboembolism, and 1 with intraoperative arrhythmia and acute cardiac infarction in VATS patients; 1 with pneumonia and 2 with acute thromboembolism in MASS patients. The incidence of approach-related complications was significantly higher in VATS patients than MASS patients $(p=0.011)$. There was no significant difference in the prevalence of pulmonary infection or iatrogenic cardiovascular injury between both surgical procedures.

The overall conversion rate from MIS to standard thoracotomy in VATS patients was $2 \%(n=15)$ and $0 \%$ in MASS patients $(p=0.001)$ (Table 4$)$. The most common cause for unplanned conversion to standard open thoracotomy was severe intrathoracic adhesion (40\%), followed by iatrogenic cardiovascular injury (20\%) and excessive uncontrollable bleeding from cancellous bone or soft tissue (20\%).

\section{Discussion}

VATS and MASS are well-known MIS methods for anterior spinal surgeries [1]. It is generally believed that using VATS for spinal surgery entails a learning curve more difficult to negotiate than does using MASS [19]; however, few studies focus on analyzing the advantages and disadvantages of using VATS and MASS to treat anterior spinal disorders. In this study, VATS required longer operating time and a higher incidence of conversion to standard open thoracotomy than did MASS at our hospital. Similarly, our review of the VATS and MASS literature for anterior T and TLJ spinal reconstruction showed that VATS was more likely to need operating time and to increase blood loss. In addition, Molina et al. [32], in a systematic review of MIS in the management of metastatic spine disease, reported that VATS was associated with longer operating time, a longer length of stay in the hospital, and more blood loss than was MASS. We found that since MASS seems more familiar to most surgeons it yields faster and safer decompression, stabilization, and reconstruction than does VATS.

We found that the overall MIS complication rate for anterior T and TLJ spinal reconstruction in the 1211 patients analyzed in the selected articles and in our hospital was $16.2 \%$ : 126 perioperative complications in VATS patients (17\%) and 71 complications in MASS patients (15\%). VATS and MASS patients had similar minor and major complication rates; however, VATS is more associated with approach-related complications. Consistent with the results of previous case series [3, 16, 19, 33], approach-related complications are most common in patients undergoing VATS. This might be true because trocar placement sometimes injures an intercostal nerve or pleural membrane, which leads to intercostal neuralgia, pleural effusion, pneumothorax, or subcutaneous emphysema [15]. Hence, the first thoracoscopic portal, which is not made using endoscopic visualization, is created using a minithoracotomy to make a $1.5 \mathrm{~cm}$ skin incision that precludes blind trocar insertion $[16,34,35]$.

Conversion to standard open thoracotomy occurred more frequently in VATS patients than in MASS patients in our hospital and in the selected literature. Consistent with our findings, other studies [35, 36] have reported that VATS is restricted because of severe pleural adhesion, poor tolerance of one-lung ventilation, and difficulty with 
TABLE 3: A summary of perioperative complications in MIS for anterior T and TLJ spinal surgery.

\begin{tabular}{|c|c|c|c|}
\hline & VATS $(n=736)$ & MASS $(n=475)$ & $p$ \\
\hline \multicolumn{4}{|l|}{ Number of patients } \\
\hline Complications in authors' institute & 27 & 11 & 0.263 \\
\hline Complications in review articles & 99 & 60 & \\
\hline A total number of complications & $126(17)$ & $71(15)$ & 0.317 \\
\hline No complication & 610 & 404 & 0.567 \\
\hline Minor complication & 102 & 59 & \\
\hline Major complication & 24 & 12 & \\
\hline Minor complication & $102(80)$ & $59(83)$ & 0.708 \\
\hline Pleural effusion, pneumothorax, and intercostal neuralgia & 52 & 18 & \\
\hline Superficial wound infection & 12 & 3 & \\
\hline Incidental durotomy & 8 & 15 & \\
\hline Pulmonary infection s/p medical treatment & 8 & 3 & \\
\hline Lung atelectasis or poor pulmonary function & 7 & 4 & \\
\hline Hypesthesia or transient motor dysfunction & 3 & 5 & \\
\hline Paralytic ileus & 0 & 5 & \\
\hline Laceration of lung parenchyma s/p repair & 4 & 0 & \\
\hline Deep vein thrombosis & 0 & 4 & \\
\hline Pharyngeal pain & 3 & 0 & \\
\hline Subcutaneous emphysema & 2 & 0 & \\
\hline Implant malposition & 1 & 1 & \\
\hline Splenic contusion & 1 & 0 & \\
\hline Iatrogenic rib fracture & 1 & 0 & \\
\hline Urinary tract Infection & 0 & 1 & \\
\hline Major complication & $24(20)$ & $12(17)$ & \\
\hline Revision & 11 & 8 & \\
\hline Graft dislodgment or implant failure or pseudoarthrosis & 7 & 5 & \\
\hline Incomplete decompression (residual disc herniation) & 3 & 2 & \\
\hline Wrong level & 1 & 0 & \\
\hline Dehiscent muscular layers in the flank & 0 & 1 & \\
\hline Pneumonia with requiring intubation & 4 & 0 & \\
\hline Iatrogenic cardiovascular injury & 3 & 0 & \\
\hline Deep wound infection & 1 & 1 & \\
\hline Permanent neurogenic deterioration & 1 & 0 & \\
\hline Postoperative acute myocardial infarction & 1 & 0 & \\
\hline Death & 3 & 3 & \\
\hline Pneumonia & 1 & 1 & \\
\hline Intraoperative arrhythmia & 1 & 0 & \\
\hline Acute thromboembolism & 1 & 2 & \\
\hline \multicolumn{4}{|l|}{ Specific complications in MIS for anterior T and TLJ spinal Surgery } \\
\hline Approach-associated complications & 54 & 18 & $0.011^{*}$ \\
\hline Pulmonary infections & 13 & 4 & 0.218 \\
\hline Iatrogenic cardiovascular injury & 3 & 0 & 0.284 \\
\hline
\end{tabular}

Data are expressed as mean \pm standard deviation or number $(\%) .{ }^{*} p<0.05$.

MIS: minimally invasive surgery; VATS: video-assisted thoracoscopic surgery; MASS: minimal access spinal surgery; T: thoracic; TLJ: thoracolumbar junction.

endoscopic control of bleeding. In addition, the conversion rate from VATS to standard open thoracotomy was $7.2 \%$ in our hospital and $1.1 \%$ in the selected articles. Metastatic vertebral tumors and infectious spondylitis occurred in most of our VATS patients whereas vertebral fracture and herniation of intervertebral disc were the majority of spinal disorders in VATS patients from the selected articles. Chronic inflammation, infection, and metastatic tumor are wellknown causes of intrathoracic adhesion [37], which might explain the relatively higher incidence of conversion to open thoracotomy in our VATS patients than in the patients in the reviewed literature. Severe pleural adhesion encountered in 
TABLE 4: Causes of conversion thoracotomy in MIS for anterior spinal surgery.

\begin{tabular}{lccc}
\hline & VATS $(n=736)$ & MASS $(n=475)$ \\
\hline Conversion to standard open procedure & $15(2)$ & 0 & 0 \\
Severe intrathoracic adhesion & 6 & 0 & $\mathbf{0 . 0 0 1}^{*}$ \\
Iatrogenic cardiovascular injury & 3 & 0 \\
Excessive uncontrollable bleeding & 3 & 0 \\
Poor tolerance of one-lung ventilation & 2 & 0 \\
Extremely narrow intercostal space & 1 & 0 \\
\hline
\end{tabular}

Data are expressed as mean \pm standard deviation or number $(\%) .{ }^{*} p<0.05$.

MIS: minimally invasive surgery; VATS: video-assisted thoracoscopic surgery; MASS: minimal access spinal surgery.

metastatic chronically infected diseases of the thoracic spine, but thoracoscopic adhesiolysis is a technically demanding procedure that must be done by an expert thoracic surgeon. Besides, intraoperative bleeding was more directly and easily controlled using cauterization, hemoclips, or suture ligation in MASS. Therefore, MASS is a reasonable MIS method for treating anterior T and TLJ spinal reconstruction and fusion, especially for metastatic and infectious spinal diseases.

This study has some limitations. First, this is a retrospective study. To minimize the statistical bias, we included patients who had undergone MIS spinal surgery done by the same surgeon (T. J. Huang) at our hospital. Second, the evidence level of the systemic review in this study is low. This is because there is still a paucity of reports that a meta-analysis needs, those that show comparative data of VATS and MASS for treating anterior spinal diseases. Additional comparative VATS and MASS studies that focus on treating anterior spinal diseases are required.

In conclusion, VATS and MASS are effective MIS methods for treating anterior T and TLJ spinal reconstruction, and they have equivalent complication rates. MASS requires less operating time and has fewer approach-related complications. VATS is more likely to have a higher conversion rate from MIS to standard open thoracotomy when severe pleural adhesion and difficulty in endoscopically controlling bleeding are encountered.

\section{Competing Interests}

All authors declare that they have no conflict of interests regarding the publication of this paper.

\section{Acknowledgments}

The authors thank Chia-Hao Chang, Ph.D., who is specialized in statistics, for statistical assistance. The authors also thank the Research Committee of Chang Gung Memorial Hospital, Taiwan, for assistance (no. CMRPG6F0131) in the Spine Operation Registry.

\section{References}

[1] O. Ofluoglu, "Minimally invasive management of spinal metastases," Orthopedic Clinics of North America, vol. 40, no. 1, pp. 155-168, 2009.
[2] M. J. Mack, J. J. Regan, W. P. Bobechko, and T. E. Acuff, "Application of thoracoscopy for diseases of the spine," The Annals of Thoracic Surgery, vol. 56, no. 3, pp. 736-738, 1993.

[3] M. J. Mack, J. J. Regan, P. C. McAfee, G. Picetti, A. Ben-Yishay, and T. E. Acuff, "Video-assisted thoracic surgery for the anterior approach to the thoracic spine," The Annals of Thoracic Surgery, vol. 59, no. 5, pp. 1100-1106, 1995.

[4] J. J. Regan, M. J. Mack, and G. D. Picetti, "A technical report on video-assisted thoracoscopy in thoracic spinal surgery: preliminary description," Spine, vol. 20, no. 7, pp. 831-837, 1995.

[5] H. M. Mayer, "A new microsurgical technique for minimally invasive anterior lumbar interbody fusion," Spine, vol. 22, no. 6, pp. 691-700, 1997.

[6] T. Kossmann, D. Jacobi, and O. Trentz, "The use of a retractor system (SynFrame) for open, minimal invasive reconstruction of the anterior column of the thoracic and lumbar spine," European Spine Journal, vol. 10, no. 5, pp. 396-402, 2001.

[7] T.-J. Huang, R. W.-W. Hsu, H.-P. Liu, Y.-S. Liao, and H.-N. Shih, "Technique of video-assisted thoracoscopic surgery for the spine: new approach," World Journal of Surgery, vol. 21, no. 4, pp. 358-362, 1997.

[8] T. J. Huang, R. W. W. Hsu, H. P. Liu et al., "Video-assisted thoracoscopic treatment of spinal lesions in the thoracolumbar junction," Surgical Endoscopy, vol. 11, no. 12, pp. 1189-1193, 1997.

[9] T.-J. Huang, R. W.-W. Hsu, H.-P. Liu, Y.-S. Liao, K.-Y. Hsu, and H.-N. Shih, "Analysis of techniques for video-assisted thoracoscopic internal fixation of the spine," Archives of Orthopaedic and Trauma Surgery, vol. 117, no. 1-2, pp. 92-95, 1998.

[10] T.-J. Huang, R. W.-W. Hsu, H.-P. Liu et al., "Video-assisted thoracoscopic surgery to the upper thoracic spine," Surgical Endoscopy, vol. 13, no. 2, pp. 123-126, 1999.

[11] T. J. Huang, R. W. Hsu, S. H. Chen, and Y. Y. Lee, "Minimal access surgery in managing anterior lumbar disorders," Clinical Orthopaedics and Related Research, no. 387, pp. 140-147, 2001.

[12] T.-J. Huang, R. W.-W. Hsu, Y.-Y. Li, and C.-C. Cheng, "Minimal access spinal surgery (MASS) in treating thoracic spine metastasis," Spine, vol. 31, no. 16, pp. 1860-1863, 2006.

[13] T.-J. Huang, R. W.-W. Hsu, S.-H. Chen, and H.-P. Liu, "Video-assisted thoracoscopic surgery in managing tuberculous spondylitis," Clinical Orthopaedics and Related Research, no. 379, pp. 143-153, 2000.

[14] C.-Y. Lee, T.-J. Huang, Y.-Y. Li, C.-C. Cheng, and M.-H. Wu, "Comparison of minimal access and traditional anterior spinal surgery in managing infectious spondylitis: a minimum 2-year follow-up," Spine Journal, vol. 14, no. 7, pp. 1099-1105, 2014.

[15] K. M. Cheung and S. Al Ghazi, "Approach-related complications of open versus thoracoscopic anterior exposures of the thoracic 
spine," Journal of Orthopaedic Surgery, vol. 16, no. 3, pp. 343-347, 2008.

[16] D. H. Kim, T. A. Jahng, R. S. V. Balabhadra, M. Potulski, and R. Beisse, "Thoracoscopic transdiaphragmatic approach to thoracolumbar junction fractures," Spine Journal, vol. 4, no. 3, pp. 317-328, 2004.

[17] C. A. Dickman, D. Rosenthal, D. G. Karahalios et al., "Thoracic vertebrectomy and reconstruction using a microsurgical thoracoscopic approach," Neurosurgery, vol. 38, no. 2, pp. 279-293, 1996.

[18] S. K. Kapoor, P. N. Agarwal, B. K. Jain Jr., and R. Kumar, "Videoassisted thoracoscopic decompression of tubercular spondylitis: clinical evaluation," Spine, vol. 30, no. 20, pp. E605-E610, 2005.

[19] L. T. Khoo, R. Beisse, and M. Potulski, "Thoracoscopic-assisted treatment of thoracic and lumbar fractures: a series of 371 consecutive cases," Neurosurgery, vol. 51, no. 5, pp. S104-S117, 2002.

[20] J.-C. Le Huec, C. Tournier, S. Aunoble, K. Madi, and P. Leijssen, "Video-assisted treatment of thoracolumbar junction fractures using a specific distractor for reduction: prospective study of 50 cases," European Spine Journal, vol. 19, S1, pp. S27-S32, 2010.

[21] G. Lü, B. Wang, J. Li, W. Liu, and I. Cheng, "Anterior debridement and reconstruction via thoracoscopy-assisted mini-open approach for the treatment of thoracic spinal tuberculosis: minimum 5-year follow-up," European Spine Journal, vol. 21, no. 3, pp. 463-469, 2012.

[22] S. D. Wait, D. J. Fox Jr., K. J. Kenny, and C. A. Dickman, "Thoracoscopic resection of symptomatic herniated thoracic discs: clinical results in 121 patients," Spine, vol. 37, no. 1, pp. 3540, 2012.

[23] A. A. Baaj, E. Dakwar, T. V. Le et al., "Complications of the mini-open anterolateral approach to the thoracolumbar spine," Journal of Clinical Neuroscience, vol. 19, no. 9, pp. 1265-1267, 2012.

[24] V. Deviren, F. A. Kuelling, G. Poulter, and M. Pekmezci, "Minimal invasive anterolateral transthoracic transpleural approach: a novel technique for thoracic disc herniation. A review of the literature, description of a new surgical technique and experience with first 12 consecutive patients," Journal of Spinal Disorders and Techniques, vol. 24, no. 5, pp. E40-E48, 2011.

[25] H. El Saghir, "Extracoelomic mini approach for anterior reconstructive surgery of the thoracolumbar area," Neurosurgery, vol. 51, no. 5, supplement, pp. S118-S122, 2002.

[26] S. N. Khan, T. Cha, J. A. Hoskins, M. Pelton, and K. Singh, "Minimally invasive thoracolumbar corpectomy and reconstruction," Orthopedics, vol. 35, no. 1, pp. e74-e79, 2012.

[27] M. Payer and C. Sottas, "Mini-open anterior approach for corpectomy in the thoracolumbar spine," Surgical Neurology, vol. 69, no. 1, pp. 25-31, 2008.

[28] K.-M. Scheufler, “Technique and clinical results of minimally invasive reconstruction and stabilization of the thoracic and thoracolumbar spine with expandable cages and ventrolateral plate fixation," Neurosurgery, vol. 61, no. 4, pp. 798-808, 2007.

[29] W. D. Smith, E. Dakwar, T. V. Le, G. Christian, S. Serrano, and J. S. Uribe, "Minimally invasive surgery for traumatic spinal pathologies: a mini-open, lateral approach in the thoracic and lumbar spine," Spine, vol. 35, pp. S338-S346, 2010.

[30] J. S. Uribe, E. Dakwar, T. V. Le, G. Christian, S. Serrano, and W. D. Smith, "Minimally invasive surgery treatment for thoracic spine tumor removal: a mini-open, lateral approach," Spine, vol. 35, no. 26S, pp. S347-S354, 2010.
[31] J. S. Uribe, W. D. Smith, L. Pimenta et al., "Minimally invasive lateral approach for symptomatic thoracic disc herniation: initial multicenter clinical experience: clinical article," Journal of Neurosurgery: Spine, vol. 16, no. 3, pp. 264-279, 2012.

[32] C. A. Molina, Z. L. Gokaslan, and D. M. Sciubba, "A systematic review of the current role of minimally invasive spine surgery in the management of metastatic spine disease," International Journal of Surgical Oncology, vol. 2011, Article ID 598148, 9 pages, 2011.

[33] A. Imperatori, N. Rotolo, M. Gatti et al., "Peri-operative complications of video-assisted thoracoscopic surgery (VATS)," International Journal of Surgery, vol. 6, no. 1, pp. S78-S81, 2008.

[34] P. C. McAfee, J. R. Regan, T. Zdeblick et al., “The incidence of complications in endoscopic anterior thoracolumbar spinal reconstructive surgery. A prospective multicenter study comprising the first 100 consecutive cases," Spine, vol. 20, no. 14, pp. 1624-1632, 1995.

[35] M. J. Perez-Cruet, R. G. Fessler, and N. I. Perin, "Review: complications of minimally invasive spinal surgery," Neurosurgery, vol. 51, no. 5, pp. S26-S36, 2002.

[36] P. Latham and K. K. Dullye, "Complications of thoracoscopy," Anesthesiology Clinics of North America, vol. 19, no. 1, pp. 187200, 2001.

[37] J. T. Huggins and S. A. Sahn, "Causes and management of pleural fibrosis," Respirology, vol. 9, no. 4, pp. 441-447, 2004. 


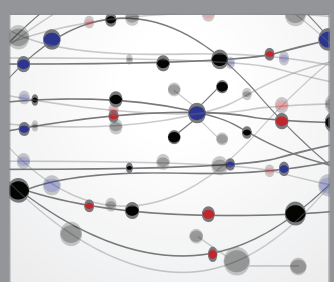

The Scientific World Journal
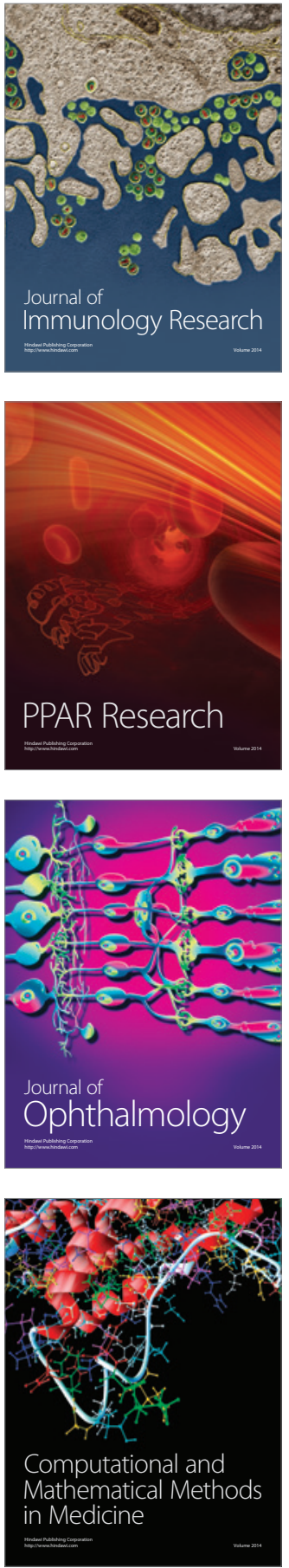

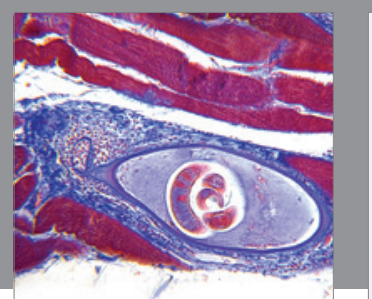

Gastroenterology Research and Practice

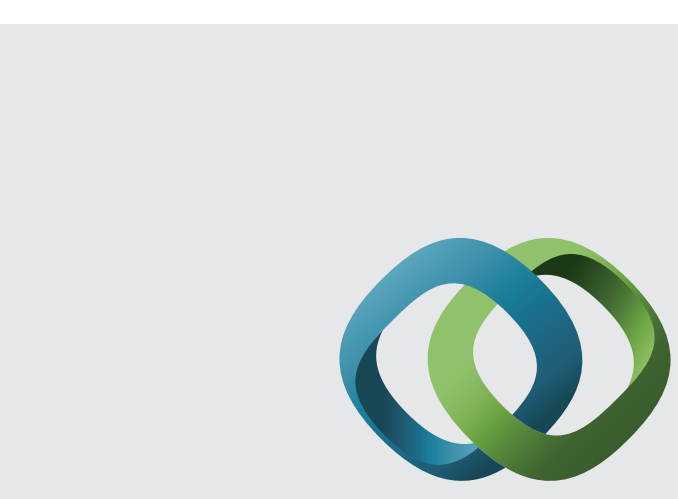

\section{Hindawi}

Submit your manuscripts at

http://www.hindawi.com
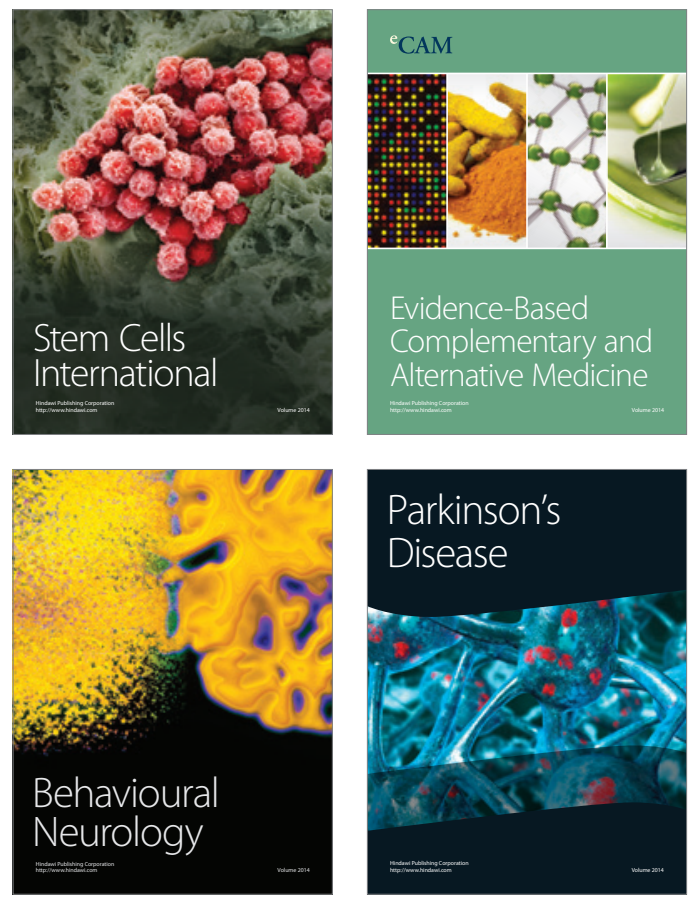
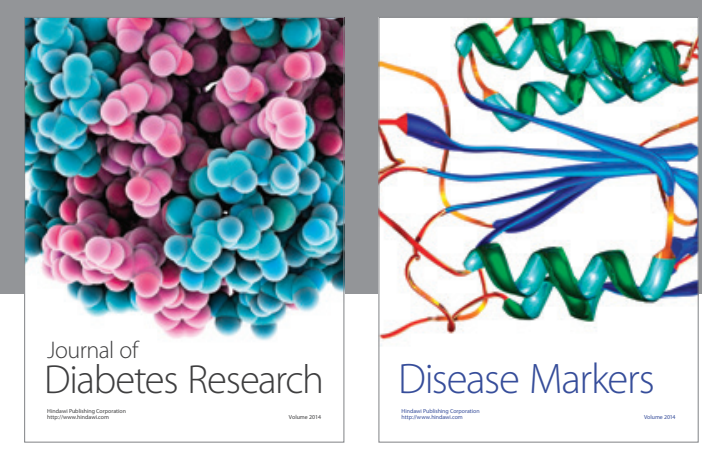

Disease Markers
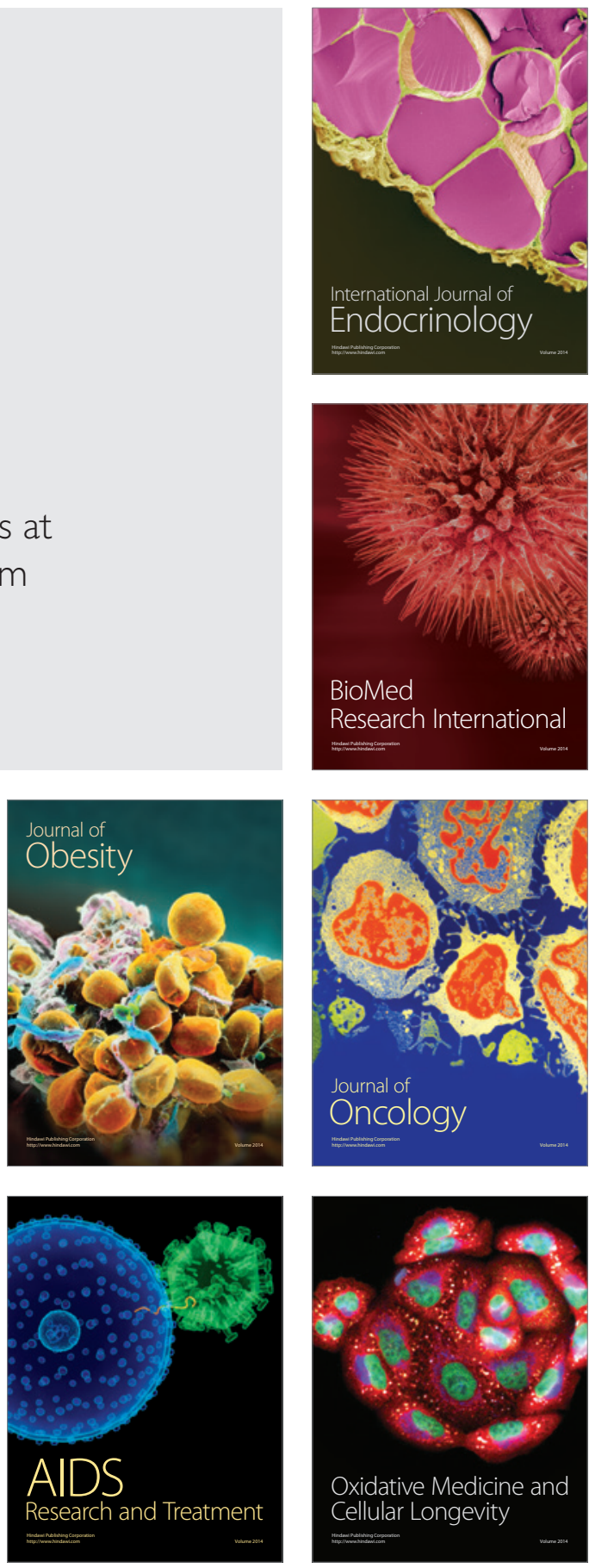